

\title{
A RELAÇÃO ENTRE AS CARACTERÍSTICAS DA EQUIPE DO ALTO ESCALÃO E A ESTRUTURA DE CAPITAL DAS EMPRESAS DE ENERGIA
}

\author{
THE RELATIONSHIP BETWEEN THE CHARACTERISTICS OF THE HIGH-RANKING \\ TEAM AND THE CAPITAL STRUCTURE OF ENERGY COMPANIES
}

\section{iD Paula de Souza Michelon ${ }^{1}$ \\ iD Rogério João Lunkes ${ }^{2}$ \\ (D) Antonio Cezar Bornia ${ }^{3}$}

\author{
${ }^{1}$ Doutora \\ Instituto Federal de Santa Catarina - IFSC \\ pauladesouza1604@gmail.com \\ 2 Pós-doutor \\ Universidade Federal de Santa Catarina - UFSC \\ rogeriolunkes@hotmail.com \\ ${ }^{2}$ Doutor \\ Universidade Federal de Santa Catarina - UFSC \\ cezar.bornia@gmail.com
}

Recebido em: 08 jun. 2019

Aprovado em: 18 dez. 2019
Resumo: A Teoria dos Escalões Superiores (TES) pondera que os valores, experiências e personalidades da equipe de gestão do topo interferem nas escolhas estratégicas da organização. Assim, espera-se que diferentes gestores escolham estruturas de capital que melhor atendam às necessidades de diferentes estratégias. Com base na TES, a presente pesquisa objetiva estudar a relação entre as características da equipe do alto escalão e a estrutura de capital das empresas de energia. Pretende-se explorar o efeito das características dos gestores no nível de endividamento da empresa, ou seja, capital de terceiros. A regressão linear de três modelos permitiu constatar que, dentre as variáveis da equipe consideradas, tempo médio no cargo foi aquela que apresentou relação estatística significativa com o nível de capital de terceiros da empresa.

Palavras-chave: Equipe do alto escalão. Estrutura de capital. Custo do capital. Capital de terceiros.

Abstract: The Upper Echelons Theory (UET) considers that the values, experiences and personalities of the top management team interfere with the organization's strategic choices. Thus, different managers are expected to choose capital structures that best meet the needs of different strategies. Based on the UET, the present research aims to study the relationship between the characteristics of the high-ranking team and the capital structure of energy companies. The intention is to explore the effect of the characteristics of managers on the level of indebtedness of the company, that is, third-party capital. The linear regression of three models showed that among the variables of the team considered, mean time in the position was that which presented a statistically significant relationship with the level of third-party capital of the company.

Keywords: Top management team. Capital structure. Cost of capital. Third-party capital. 


\section{Introdução}

Para entender por que as organizações fazem o que fazem ou têm o desempenho que têm, devem-se considerar os vieses e as disposições de seus atores mais poderosos - os executivos do alto escalão (Hambrick \& Mason, 1984). As organizações refletem o que seus líderes pensam, sentem, percebem e acreditam (Oppong, 2014).

Li (2016) sustenta que a experiência e o conhecimento das equipes de gestão do topo podem criar recursos e capacidades estratégicas para toda a empresa. Essas aptidões desempenham um papel crucial na alocação de recursos, afetando, por seu turno, as taxas de crescimento (Hutzschenreuter \& Horstkotte, 2013).

Nesse sentido, a Teoria dos Escalões Superiores (TES) argumenta que os valores, experiências e personalidades da equipe de gestão do topo interferem nas escolhas estratégicas da organização, bem como no sucesso dessas escolhas (Hambrick, 2007). Características demográficas, como idade, sexo, educação e experiência funcional são indicativos de aspectos gerenciais cognitivos e afetivos subjacentes que determinam as decisões das equipes de gestão, que afetam posteriormente o desempenho da organização (Bell, Villado, Lukasik, Belau, \& Briggs, 2007).

Dentre as perspectivas da referida Teoria, Hambrick (2007) afirma que as equipes de escalões superiores heterogêneas, compostas por gerentes com diferentes habilidades e perfis demográficos, podem explicar as diferenças nas escolhas estratégicas, inovação e desempenho de empresas.

A base cognitiva e os valores dos executivos dos escalões superiores estão em função de suas características observáveis como idade, tempo no cargo, educação, raízes socioeconômicas e situação financeira. Por conseguinte, os resultados organizacionais estão associados com as características observáveis desses profissionais (Carpenter, Geletkanycz, \& Sanders, 2004).

Bertrand e Schoar (2003) argumentam que os gestores desempenham um papel crítico nas políticas de investimento, nas políticas financeiras, nas estratégias organizacionais e no desempenho operacional da empresa. Os gestores de escalões superiores influenciam importantes decisões estratégicas relacionadas a atividades como investimentos, formação de alianças estratégicas e internacionalização, sendo que cada uma delas provocará impactos no desempenho (Carpenter \& Fredrickson, 2001; Cabrera-Suárez \& Martín-Santana, 2013, Wang, 2015; Xie, Wang, \& Qi, 2015).

Uma suposição crítica de teorias de gestão estratégica é que, em qualquer indústria, os potenciais consumidores são heterogêneos em relação a seus gostos e preferências. Então, se a estratégia competitiva orienta decisões de investimento da empresa (Chandler, 1962) e a escolha de investimentos pode influenciar a escolha de financiamento (Williamson,1988), então deve-se esperar que diferentes estruturas de capital melhor atendem às necessidades de diferentes estratégias ( $O^{\prime}$ Brien, 2003). 
Chevalier e Scharfstein (1995) e Maksimovic (1998) procuraram esclarecer a relação entre estratégia e estrutura de capital, mas sem verificar a influência das características dos gestores. Barker e Mueller (2002), Kor (2006) e Chen, Hsu e Huang (2010) estudaram a conexão entre as características dos executivos e a estrutura de capital das empresas, mas com foco no financiamento com pesquisa e desenvolvimento.

Nesse contexto, a presente pesquisa objetiva estudar a relação entre as características da equipe do alto escalão e a estrutura de capital das empresas de energia. Pretende-se explorar o efeito das características dos gestores no nível de endividamento da empresa, ou seja, capital de terceiros.

Justifica-se a escolha por empresas do setor de energia elétrica pelo papel fundamental que o setor tem na atividade econômica: mútua interdependência entre a economia e o setor elétrico. Isso porque o setor de energia utiliza insumos de vários setores produtivos e este setor também constitui insumo para outros diversos tipos de atividades econômicas (Bhattacharyya, 2007; Pereira, Santana, Mendes, \& Khan, 2008). Por conta desse papel estratégico do setor, é relevante entender como as características dos gestores influenciam na estrutura de capital das empresas.

\section{Revisão teórica}

\subsection{Teoria dos Escalões Superiores - TES}

A tomada de decisão é um elo importante na administração das empresas. A gestão das empresas é construída com as decisões de diferentes níveis, sejam eles de grande importância ou não. A rapidez, a precisão e a execução da tomada de decisão afetam diretamente a qualidade da decisão que, de certa forma, tem grande influência sobre o efeito da gestão das empresas e sobre a eficiência da equipe (Guo, Liu, \& Yang, 2011).

A Teoria dos Escalões Superiores (TES), difundida por Hambrick e Mason (1984), sugere que os resultados e processos estratégicos organizacionais são uma função das características gerenciais dos gestores superiores ou gestores de topo. A principal noção da Teoria é que as escolhas estratégicas, ao contrário das decisões operacionais, são mais oriundas de fatores comportamentais do que de cálculo mecânico para a otimização econômica. Como decorrência, as escolhas estratégicas geralmente possuem uma grande quantidade de componentes comportamentais e, em alguma medida, refletem as idiossincrasias dos tomadores de decisão. As idiossincrasias dos gestores de topo incluem sua base cognitiva (conhecimento/suposição sobre eventos futuros, conhecimento de alternativas e conhecimento de consequências de alternativas) e valores (princípios para ordenar consequências ou alternativas). Essas idiossincrasias filtram e enquadram a situação de decisão que os executivos enfrentam e, eventualmente, criam suas percepções da situação (Chuang, Nakatani, \& Zhou, 2007; Chuang, Nakatani, \& Zhou, 2009). 
A TES tem duas principais afirmações teóricas (Hambrick \& Mason, 1984; Carpenter et al., 2004; Hambrick, 2007):

i. as escolhas estratégicas e os resultados organizacionais dos executivos são influenciados por seus valores e base cognitiva; e

ii. esses valores e bases cognitivas estão em função de características observáveis, tais como idade, experiência funcional, nível educacional, raízes socioeconômicas, posição financeira e características de grupo.

De acordo com a TES, a equipe de escalões superiores, e não os indivíduos, são os principais tomadores de decisão dentro de uma organização. A premissa da Teoria é que o foco sobre as características da equipe irá produzir explicações mais fortes de resultados organizacionais do que o foco habitual no alto executivo individual (por exemplo, CEO). Isso porque, liderança de uma organização complexa é uma atividade compartilhada, e as cognições, capacidades e interações de toda a equipe afetam o comportamento estratégico (HAmbrick, 2007).

A definição de top management team ou equipe de gestão de topo (EGT) baseia-se no trabalho original de Cyert e March (1963), que se referiram a ela ao tratar da coalizão dominante (Carpenter et al., 2004). Em um nível mais prático, o estudo de toda uma equipe aumenta a força potencial da teoria para prever, porque o chefe executivo compartilha tarefas e, em certa medida, o poder com outros membros da equipe (Hambrick \& Mason, 1984).

A TES considera que a base cognitiva, os valores e a percepção dos gestores são características inobserváveis. Dessa maneira, as características gerenciais mensuráveis podem ser substitutas adequadas e capazes de fornecer indicadores razoáveis dessas construções latentes (Carpenter et al., 2004).

Hambrick e Mason (1984) elaboraram uma lista não esgotada de características gerenciais observáveis, incluindo idade, experiências funcionais, experiências de carreira, educação, raízes socioeconômicas, posição financeira e características de grupo. Do mesmo modo, indicaram 21 proposições relacionando essas características às escolhas estratégicas e ao desempenho dos resultados organizacionais. Tais proposições foram categorizadas em sete grupos: faixa etária relacionada à idade, outras experiências de carreira, educação formal, contexto socioeconômico, situação financeira e heterogeneidade de grupo (Chuang et al., 2007).

Assim, estudos que levam em conta os níveis médios da equipe sugerem que as principais características executivas, como a idade (Goll, Johnson \& Rasheed, 2008; Koufopoulos, Zoumbos, Argyropoulou, \& Motwani, 2008), nível educacional (Díaz-Fernández, González-Rodríguez, \& Pawlak, 2014; Díaz-Fernández, González-Rodríguez, \& Simonetti, 2015), tempo no cargo (Pegels \& Yang, 2000; 
Gu, 2008), e a heterogeneidade (Naranjo-Gil, Hartmann, \& Maas, 2008; Li, Zhang, \& Zhang, 2015) estão associadas a decisões e resultados estratégicos.

\subsection{Estrutura de capital}

\subsubsection{Teorias da estrutura de capital}

A estrutura de capital de uma empresa representa a maneira como está composto seu passivo. O tema é marcado por diversas teorias, cujas principais são Modigliani e Miller, Trade-off e Pecking Order.

A Teoria de Modigliani e Miller almeja elucidar os determinantes que influenciam a estrutura de capital das empresas, utilizando-se da aplicação do conceito de equilíbrio de mercado de arbitragem, em que o valor de mercado de uma empresa é independente da sua estrutura de capital. Para Modigliani e Miller (1958) empresas idênticas possuem o mesmo valor independentemente do sua estrutura de financiamento é baseada em capitais próprios ou de terceiros. Sendo assim, a estrutura de capital não era determinante no valor da empresa e a decisão de investimento independente da origem dos recursos de terceiros.

A Teoria do trade-off aponta para a existência de uma estrutura ótima de capital, mas não é capaz de explica a correlação entre alta lucratividade e baixos índices de endividamento. Não adianta dizer (sem mais explicações) que os gerentes são "excessivamente conservadores" ou "não maximizam o valor". Culpar os gerentes, em vez dos economistas, pelo fracasso da teoria dos economistas. Além disso, um exame das táticas de financiamento rapidamente descarta a ideia de que os gerentes não prestam atenção aos impostos (Myers, 2001).

Na Teoria de Pecking Order a estrutura de capital é resultado de decisões hierárquicas com relação à captação de recursos para cobrir os investimentos da empresa. Myers e Majluf (1984) e Myers (1984) assumiram que os gestores agem no interesse dos acionistas existentes e se recusam a emitir ações subvalorizadas a menos que a transferência para novos acionistas seja compensada pelo valor presente líquido da oportunidade de crescimento. Isso leva a um equilíbrio comum no qual as empresas podem emitir ações, mas apenas a um preço reduzido.

Os gestores otimistas, que confiam que as ações de suas empresas estão subvalorizadas, terão a oportunidade de emitir dívida em vez de capital próprio. Mas não necessariamente apenas gestores pessimistas vão querer emitir igualdade. Se a dívida é uma alternativa aberta, então qualquer tentativa de vender ações pode revelar que não é uma boa opção de compra. Logo, as emissões de ações serão rejeitadas pelos investidores se a dívida estiver disponível em termos justos e, em equilíbrio, somente a dívida será emitida. As questões de igualdade acontecerão apenas quando a dívida for onerosa. Nesse 
caso, até gestores otimistas podem recorrer ao mercado de ações para obter financiamento (Myers \& Majluf, 1984).

\subsubsection{Indicadores de Estrutura de Capital}

Na estrutura de capital o cálculo das taxas de desconto envolve, dentre outros, o Custo da Dívida (CD), o Custo do Capital Próprio (CCP) e o Custo Médio Ponderado do Capital (CMPC).

O CD ou custo do capital de terceiros é o custo atual após o imposto de renda do levantamento de fundos de longo prazo, por meio de empréstimos (Gitman, 2010). É a taxa de desconto que avalia o custo atual para a empresa de contrair fundos para financiamento de projetos (Damodaran, 2012).

Damodaran (2012), por seu turno, salienta que o CD não é a taxa do cupom dos bônus que a empresa tem a pagar, nem a taxa a qual a empresa conseguiu tomar um financiamento no passado. Ainda que essas possam auxiliar na determinação do custo dos juros que a empresa terá de pagar, não definem o CD após o pagamento de impostos. Por conseguinte, uma empresa que contabilizou uma dívida contraída quando as taxas de juros estavam baixas, não pode declarar que tem um CD baixo se o nível geral das taxas de juros ou seu risco de inadimplência aumentou no período.

Pelo fator temporal, os financiamentos podem ser de curto ou de longo prazo. Prazo maior implica risco maior. No caso das dívidas de curto prazo, os valores contábeis e os valores de mercado tendem a ser próximos, o que possibilita utilizar os próprios valores contábeis como equivalentes a seus valores de mercado (Ross, 2007).

O CCP ou custo do patrimônio líquido é a taxa de retorno que os investidores exigem para realizar um investimento patrimonial em uma empresa, retorno este que poderia ser obtido em outras aplicações de mesmo risco de mercado (Damodaran, 2012).

De acordo com Ross (2007), o CCP é de mensuração mais difícil, pois não existe um modo de constatar diretamente o retorno exigido pelos investidores em ações de uma empresa. A estimativa do CCP é feita com base em modelos sugeridos na literatura de finanças.

O modelo de avaliação com crescimento constante (modelo de Gordon) e o modelo de formação de preços de ativos (MFPA) são exemplo para medir o CCP (Gitman, 2010).

O CMPC é a taxa de retorno requerida em qualquer proposta de investimento que possui o mesmo nível de risco que os ativos existentes da empresa. Deve ser ajustado para mais ou para menos, a depender do tipo de projeto (por exemplo, projetos de substituição são de menor risco, enquanto de expansão ou novos são de risco mais elevado) ou para diferentes unidades organizacionais (Bennouna, Meredith, \& Marchant, 2010).

De maneira simplificada, o CMPC pode ser definido como a média ponderada dos custos dos diversos componentes de financiamento, abarcando dívida e patrimônio líquido, utilizados por uma 
empresa para financiar suas necessidades financeiras (Damodaran, 2012). Calcula-se multiplicando o custo específico de cada modalidade de financiamento por sua participação na estrutura de capital da empresa e, por fim, somam-se os valores ponderados (Gitman, 2010).

Via de regra, sugere-se que as empresas apliquem taxas diferenciadas para os projetos de investimentos, unidades ou divisões organizacionais. Ao analisar o retorno de mercado, a empresa pode desenvolver taxas distintas para os diversos investimentos, até mesmo para projetos fora do seu negócio principal (Damodaran, 2012).

\section{Procedimentos metodológicos}

\subsection{Hipóteses da pesquisa}

O nível médio de idade da Equipe de Gestão de Topo (EGT) foi proposto para ser associada a tendências como receptividade à mudança, vontade de adotar novas ideias, busca de novidade, tomada de risco e flexibilidade. Tais predisposições, por sua vez, estão associadas à inovação estratégica, à extensão da diversificação das empresas, ao grau de firme expansão internacional, ao crescimento da empresa, entre outros. O argumento subjacente é que à medida que a idade média da EGT aumenta, a equipe se torna mais resistente à tomada de risco e mudança (Acar, 2016).

Gerentes mais jovens podem ter menos compromisso com o status quo e, portanto, podem estar mais dispostos a empreender estratégias novas e sem precedentes (Hambrick \& Mason, 1984). Devido ao compromisso com o status quo, uma EGT mais antiga pode ser mais relutante em tentar novas ideias ou assumir riscos (Koufopoulos et al., 2008). Por outro lado, os gestores mais velhos já estabeleceram suas redes sociais, estilos de vida e planejamento de carreiras e tenderam a se tornar aversivos ao risco (Chuang et al., 2009).

Equipes mais antigas com a propensão a ser avessos ao risco e com segurança financeira e profissional preocupações tenderiam a escolher uma forma mais conservadora estrutura de capital, enquanto as EGTs mais jovens mais dispostos a usar capital de terceiros (Chen et al., 2010). Dessa maneira, constrói-se a seguinte hipótese:

H1: A idade média dos gestores está positivamente relacionada com o nível de capital de terceiros das empresas.

As experiências observáveis dos gestores são proxies válidos para sua orientação cognitiva, valores e conhecimentos que posteriormente impactarão as escolhas estratégicas. Gestores com mais experiências internacionais, educação e experiência funcional diversificada tomam decisões para minimizar os riscos se comparados a gestores com menos experiência e educação ou experiência 
funcional similar. Os gestores de topo que possuem tais características tomam decisões estratégicas eficazes, como a avaliação completa de múltiplas opções para novas oportunidades, minimizando riscos e incertezas (Bany-Ariffin, Mcgowan, Júnior Tunde, \& Shahnaz, 2014).

A TES propõe uma associação linear positiva entre o nível de educação dos executivos e a base de conhecimento, a complexidade cognitiva, a abertura à mudança, a tolerância para a ambiguidade e a propensão ao risco. Nível educacional mais elevado é indicativo de maior base de conhecimentos (Herremann \& Datta 2005). A maior base de conhecimento, por sua vez, deverá estar associada a estratégias mais eficazes e a uma gestão mais eficiente da empresa.

Porém, equipes mais instruídas possuem conhecimento avançado e maior capacidade cognitiva. Isso, por sua vez, poderia aumentar sua capacidade de tolerar ambiguidade, absorver novas informações e analisar, resolver e implementar soluções para problemas complexos. Mais especificamente, as EGTs com mais escolaridade seriam menos propensos a optar por uma estrutura de capital conservadora (Chen et al., 2010).

Assim, gestores com maior nível educacional podem ser mais confiantes em suas decisões de investimento e não necessitarem de tanta folga financeira se comparados aqueles com nível educacional inferior. Portanto, gerentes com maior nível de formação seriam menos propensos a optar por uma estrutura de capital conservadora para o financiamento do investimento (Bortoluzzi, Zakaria, Santos, \& Lunkes, 2016). Nesse contexto, tem-se a segunda hipótese:

H2: O nível médio educacional está negativamente relacionado com o nível de capital de terceiros das empresas.

O tempo no cargo pode representar a coesão social, a alta influência organizacional e o compromisso com o status quo, bem como a inércia e a falta de inovação (Bantel \& Jackson, 1989; Michel \& Hambrick, 1992). Assim, o tempo do cargo da EGT pode representar um compromisso com o status quo e baixa criatividade (Hoffman, Williams, Lamont, \& Geiger, 2000; Wiersema \& Bantel, 1992).

A longa duração média de tempo no cargo resulta em níveis decrescentes de comunicação global, porque os membros do grupo sentem que são capazes de antecipar os pontos de vista de outros membros e ocorre o aumento da especialização (Wiersema \& Bantel, 1992). Portanto, o longo mandato da equipe pode levar a um maior isolamento em relação a fontes externas de informação, o que pode levar aos membros a se tornarem menos receptivos à mudança e à inovação (Camelo, Fernández-Alles \& Hernández, 2010).

Gestores com menos tempo na empresa podem estar mais dispostos a assumir riscos, porque são obrigados a produzir resultados e a se mostrarem como gestores competentes. Gestores de longo prazo, por outro lado, podem ter uma abordagem mais avessa ao risco, porque são menos pressionados a se comprovarem (Kor, 2006). Com isto, propõe-se a terceira hipótese: 
H3: O tempo médio no cargo está negativamente relacionado com o nível de capital de terceiros das empresas.

\subsection{Amostra e coleta de dados}

A população do estudo é formada por 59 empresas do setor de energia elétrica cotadas na Brasil, Bolsa, Balcão $\left(B^{3}\right)$, bolsa de valores oficial do Brasil, em 20 de maio de 2019. Do total, 1 não disponibilizou as informações, perfazendo uma amostra de 58 empresas. Os dados foram coletados no endereço eletrônico da $B^{3}$ entre os dias 27 de maio a 05 de junho de 2019 referentes ao exercício de 2018.

Quadro 1 - Composição da amostra

\begin{tabular}{|c|c|c|}
\hline \multicolumn{3}{|c|}{ Empresas do Setor de Energia Elétrica } \\
\hline AES TIETE ENERGIA SA & $\begin{array}{l}\text { CIA ESTADUAL DE DISTRIB ENER } \\
\text { ELET-CEEE-D }\end{array}$ & ENGIE BRASIL ENERGIA S.A. \\
\hline $\begin{array}{l}\text { AFLUENTE TRANSMISSÃO DE } \\
\text { ENERGIA ELÉTRICA S/A }\end{array}$ & $\begin{array}{l}\text { CIA ESTADUAL } \\
\text { GER.TRANS.ENER.ELET-CEEE-GT }\end{array}$ & EQUATORIAL ENERGIA S.A. \\
\hline ALUPAR INVESTIMENTO S/A & $\begin{array}{l}\text { CIA PARANAENSE DE ENERGIA - } \\
\text { COPEL }\end{array}$ & $\begin{array}{l}\text { FERREIRA GOMES ENERGIA } \\
\text { S.A. }\end{array}$ \\
\hline AMPLA ENERGIA E SERVICOS S.A. & CIA PAULISTA DE FORCA E LUZ & $\begin{array}{l}\text { ITAPEBI GERACAO DE ENERGIA } \\
\text { S.A. }\end{array}$ \\
\hline $\begin{array}{l}\text { CACHOEIRA PAULISTA } \\
\text { TRANSMISSORA ENERGIA S.A. }\end{array}$ & CIA PIRATININGA DE FORCA E LUZ & LIGHT S.A. \\
\hline CEMIG DISTRIBUICAO S.A. & CPFL ENERGIA S.A. & $\begin{array}{l}\text { LIGHT SERVICOS DE } \\
\text { ELETRICIDADE S.A. }\end{array}$ \\
\hline $\begin{array}{l}\text { CEMIG GERACAO E TRANSMISSAO } \\
\text { S.A. }\end{array}$ & CPFL ENERGIAS RENOVÁVEIS S.A. & NEOENERGIA S.A. \\
\hline $\begin{array}{l}\text { CENTRAIS ELET BRAS S.A. - } \\
\text { ELETROBRAS }\end{array}$ & CPFL GERACAO DE ENERGIA S.A. & OMEGA GERAÇÃO S.A. \\
\hline $\begin{array}{l}\text { CENTRAIS ELET DE SANTA CATARINA } \\
\text { S.A. }\end{array}$ & $\begin{array}{l}\text { CTEEP - CIA TRANSMISSÃO ENERGIA } \\
\text { ELÉTRICA PAULISTA }\end{array}$ & $\begin{array}{l}\text { PRODUTORES ENERGET.DE } \\
\text { MANSO S.A.- PROMAN }\end{array}$ \\
\hline CENTRAIS ELET DO PARA S.A. - CELPA & EDP - ENERGIAS DO BRASIL S.A. & $\begin{array}{l}\text { REDE ENERGIA PARTICIPAÇÕES } \\
\text { S.A. }\end{array}$ \\
\hline $\begin{array}{l}\text { CESP - CIA ENERGETICA DE SAO } \\
\text { PAULO }\end{array}$ & $\begin{array}{l}\text { EDP ESPIRITO SANTO DISTRIBUIÇÃO } \\
\text { DE ENERGIA S.A. }\end{array}$ & RENOVA ENERGIA S.A. \\
\hline $\begin{array}{l}\text { CIA CELG DE PARTICIPACOES - } \\
\text { CELGPAR }\end{array}$ & $\begin{array}{l}\text { EDP SÃO PAULO DISTRIBUIÇÃO DE } \\
\text { ENERGIA S.A. }\end{array}$ & $\begin{array}{l}\text { RGE SUL DISTRIBUIDORA DE } \\
\text { ENERGIA S.A. }\end{array}$ \\
\hline $\begin{array}{l}\text { CIA ELETRICIDADE EST. DA BAHIA - } \\
\text { COELBA }\end{array}$ & ELEKTRO REDES S.A. & $\begin{array}{l}\text { RIO PARANAPANEMA ENERGIA } \\
\text { S.A. }\end{array}$ \\
\hline CIA ENERGETICA DE BRASILIA & $\begin{array}{l}\text { ELETROBRÁS PARTICIPAÇÕES S.A. - } \\
\text { ELETROPAR }\end{array}$ & SANTO ANTONIO ENERGIA S.A. \\
\hline $\begin{array}{l}\text { CIA ENERGETICA DE MINAS GERAIS - } \\
\text { CEMIG }\end{array}$ & $\begin{array}{l}\text { ELETROPAULO METROP. ELET. SAO } \\
\text { PAULO S.A. }\end{array}$ & $\begin{array}{l}\text { STATKRAFT ENERGIAS } \\
\text { RENOVAVEIS S.A. }\end{array}$ \\
\hline $\begin{array}{l}\text { CIA ENERGETICA DE PERNAMBUCO - } \\
\text { CELPE }\end{array}$ & $\begin{array}{l}\text { EMAE - EMPRESA METROP.AGUAS } \\
\text { ENERGIA S.A. }\end{array}$ & $\begin{array}{l}\text { TERMELÉTRICA PERNAMBUCO } \\
\text { III S.A. }\end{array}$ \\
\hline CIA ENERGETICA DO CEARA - COELCE & $\begin{array}{l}\text { ENERGISA MATO GROSSO DO SUL - } \\
\text { DIST DE ENERGIA S.A. }\end{array}$ & TERMOPERNAMBUCO S.A. \\
\hline $\begin{array}{l}\text { CIA ENERGETICA DO MARANHAO - } \\
\text { CEMAR }\end{array}$ & $\begin{array}{l}\text { ENERGISA MATO GROSSO- } \\
\text { DISTRIBUIDORA DE ENERGIA S/A }\end{array}$ & $\begin{array}{l}\text { TRANSMISSORA ALIANÇA DE } \\
\text { ENERGIA ELÉTRICA S.A. }\end{array}$ \\
\hline $\begin{array}{l}\text { CIA ENERGETICA DO RIO GDE NORTE } \\
\text { - COSERN }\end{array}$ & $\begin{array}{l}\text { ENERGISA S.A. } \\
\text { ENEVA S.A }\end{array}$ & UPTICK PARTICIPACOES S.A. \\
\hline
\end{tabular}

Fonte: Brasil, Bolsa, Balcão (2018). 
A coleta de dados foi realizada em duas etapas. A primeira compreendeu a coleta dos das variáveis independentes de idade, nível educacional e tempo no cargo dos gestores das empresas, por meio dos formulários de referência de 2018, na item de assembleia e administração. O Quadro 2 apresenta as variáveis independentes:

Quadro 2 - Variáveis independentes

\begin{tabular}{|c|c|c|}
\hline Variável independente & Abreviação & Forma de cálculo \\
\hline Idade média & I_IM & Logaritmo da média de idade dos gestores \\
\hline Nível médio educacional & NME & $\begin{array}{l}\text { Nível médio ou menos }=0 \text {, Graduação = 1, Pós-graduação ou } \\
\text { Master Business Administration }(M B A)=2 \text {, Mestrado }=3 \text {, } \\
\text { Doutorado }=4 .\end{array}$ \\
\hline Tempo médio no cargo & I_TMC & Logaritmo da média de meses no cargo \\
\hline
\end{tabular}

Fonte: Autores.

A segunda etapa diz respeito aos dados financeiros presentes no Balanço Patrimonial de 2018 das companhias, exigível total e patrimônio líquido, os quais foram extraídos por meio do software Economática ${ }^{\circledR}$.

A variável dependente Nível de Capital de Terceiros (NCT) é calculada pela divisão do Exigível Total pelo Patrimônio Líquido ((Passivo Circulante + Passivo Não Circulante) /Patrimônio Líquido). A seguir apresenta-se a equação do modelo:

$$
N C T=\partial+\beta_{1} \mathfrak{I}+\beta_{2} N M E+\beta_{3} T M C+\mu
$$

em que $\partial$ é a constante da função, $\beta_{1}, \beta_{2}$ e $ß_{3}$ são os coeficientes estimados da regressão e $\mu$ é o erro da regressão.

Os dados são tratados com a utilização do procedimento estatístico regressão linear múltipla em Mínimos Quadrados Ordinários (MQO). Para o tratamento dos dados utilizou-se o software Gretl. Inicialmente foi realizada a correlação linear entre as variáveis e, na sequência, a regressão múltipla.

\section{Resultados e análises dos dados}

Os resultados analisados dizem respeito a 303 pessoas de 58 empresas ou Equipes de Gestão de Topo (EGT). A média de integrantes por equipe é aproximadamente de 5,22, sendo mediana 5 , mínimo 2 e máximo 10 membros. A Tabela 1 evidencia as características dos diretores que compõem as EGTs. 
Tabela 1 - Características descritivas da amostra (58 empresas)

\begin{tabular}{|c|c|c|}
\hline \multirow[t]{2}{*}{ Variáveis } & \multicolumn{2}{|c|}{ Frequência } \\
\hline & Média & Desvio Padrão \\
\hline Idade & 49,98 & 6,35 \\
\hline Tempo no cargo & 38,51 & 28,54 \\
\hline Nível educacional & 1,75 & 0,57 \\
\hline & Frequência Relativa & Frequência \\
\hline Graduação & 131 & $43,23 \%$ \\
\hline Pós-graduação ou MBA & 111 & $36,63 \%$ \\
\hline Mestrado & 57 & $18,81 \%$ \\
\hline Doutorado & 4 & $1,32 \%$ \\
\hline
\end{tabular}

Fonte: Dados da pesquisa.

A análise descritiva permite inferir que as 58 companhias possuem gestores com média de idade de 50 anos e 3 anos de tempo no cargo. Em relação ao nível educacional, a maioria possui somente graduação ou também pós-graduação/MBA. A especialização stricto sensu ainda alcança menos de 20\% dos diretores de empresas de energia elétrica.

No que diz respeito à estrutura de capital, observou-se que em média 69,9\% são provenientes de terceiros, enquanto que 31,1\% são derivados de capital próprio. Em outras palavras, as empresas da amostra tendem a recorrer para recursos de terceiros, de modo geral, para manutenção de suas atividades.

Antes de realizar o teste das hipóteses, procede-se a verificação de valores outliers na amostra. Nesse caso, verificaram-se a existência de valores outliers de endividamento. Notou-se que 7 empresas estavam com tais valores acima ou abaixo dos limites superior e inferior da amostra. Para que tais dados não distorçam a análise dos resultados, foram eliminados, perfazendo um total de 51 observações (empresas de energia elétrica).

Realizam-se testes de multicolinearidade dos dados e correlação de Pearson para ratificar a normalidade dos dados. O Quadro 2 apresenta a matriz de correlação linear, o qual representa o grau de associação entre as variáveis. As variáveis são correlacionadas quando a mudança em uma dela implica em modificações na(s) outra(s) variável(eis). O coeficiente pode oscilar de -1(correlação negativa) a +1 (correlação positiva). Valor zero denota ausência de correlação, mais próximo a +1 significa correlação positiva forte e mais próximo a -1 correlação negativa forte. 
Michelon, P. de S., Lunkes, R. J., \& Bornia, A. C. (2021, abr./jun.). A relação entre as características da equipe do alto escalão e a estrutura de capital das empresas de energia

Tabela 2 - Matriz de correlação (51 empresas)

\begin{tabular}{l|lll|l}
\multicolumn{1}{c}{ Variáveis } & & I_IM & \multicolumn{1}{c}{ NME } & \multicolumn{1}{c}{ I_TMC } \\
\hline I_IM & 1 & $-0,0752$ & 0,1068 & $-0,0267$ \\
\hline NME & - & 1 & 0,2469 & 0,0313 \\
\hline I_TMC & - & - & 1 & 0,2860 \\
\hline NCT & - & - & - & 1
\end{tabular}

Fonte: Dados da pesquisa.

Os valores evidenciados demonstram que a associação de Logaritmo da média de idade dos gestores com Nível médio educacional e Nível de Capital de Terceiros é negativa (contrária). Ou seja, quanto maior o Logaritmo da média de idade dos gestores, menor tende a se o Nível médio educacional e Nível de Capital de Terceiros da empresa. A maior correlação é entre Logaritmo da média de meses no cargo e Nível de Capital de Terceiros (28,6\%), mas é pouco expressiva.

Para testar as hipóteses desenvolvidas na presente pesquisa, realizam-se modelos de regressão linear múltipla e simples. A Tabela 3 contempla os resultados de três modelos, os quais foram testados com o propósito de demonstrar o que melhor explica as variáveis.

Tabela 3 - Modelos de regressão linear

\begin{tabular}{|c|c|c|c|c|c|}
\hline & & & & & \\
\hline & Variável & Coeficiente & Erro padrão & Razão-t & P-valor \\
\hline Modelo 1 & const & 2,97206 & 6,57449 & 0,4521 & 0,6534 \\
\hline R-quadrado = 0,087307 & IIIM & $-0,739776$ & 1,67540 & $-0,4416$ & 0,6609 \\
\hline $\begin{array}{l}\text { R-quadrado ajustado }= \\
0,027784\end{array}$ & NME & $-0,126407$ & 0,379921 & $-0,3327$ & 0,7409 \\
\hline P-valor $(F)=0,235968$ & I_TMC & 0,549457 & 0,264332 & 2,079 & $0,0433^{* *}$ \\
\hline Modelo 2 & Variável & Coeficiente & Erro padrão & Razão-t & P-valor \\
\hline R-quadrado = 0,085111 & const & 2,59628 & 6,41519 & 0,4047 & 0,6875 \\
\hline $\begin{array}{l}\text { R-quadrado ajustado }= \\
0,046179\end{array}$ & IIIM & $-0,681029$ & 1,65024 & $-0,4127$ & 0,6817 \\
\hline P-valor $(F)=0,123640$ & I_TMC & 0,526846 & 0,253019 & 2,082 & $0,0428^{* *}$ \\
\hline Modelo 3 & Variável & Coeficiente & Erro padrão & Razão-t & P-valor \\
\hline R-quadrado = 0,081796 & const & $-0,0270600$ & 0,856348 & $-0,03160$ & 0,9749 \\
\hline $\begin{array}{l}\text { R-quadrado ajustado }= \\
0,062666\end{array}$ & I_TMC & 0,515694 & 0,249388 & 2,068 & $0,0441^{* *}$ \\
\hline
\end{tabular}

Fonte: Dados da pesquisa. 
A estimação dos três modelos foi feita apor meio de Mínimos Quadrados Ordinários. Ao analisar os coeficientes estimados para as variáveis independentes do primeiro Modelo 1, verifica-se que I_TMC (Logaritmo da média de meses no cargo) foi a variável que apresentou significância estatística a um nível de $5 \%(p$-valor $=0.0433)$. O valor do R-quadrado denota que o referido modelo explica aproximadamente $8,73 \%$ do nível de capital de terceiros da empresa.

Na estimação do Modelo 2 foi eliminada a variável Nível médio educacional, uma vez que apresentou o $p$-valor mais alto (0.7409). Com isso, houve diminuição no poder explicativo do Modelo, se comparado ao Modelo 1, uma vez que uma variável explicativa foi excluída. Mas, a significância do Modelo 2 é maior ( $p$-valor $=0,123640)$.

No mesmo raciocínio, eliminou-se no Modelo 3 a variável Logaritmo da média de idade dos gestores por não apresentar significância estatística no Modelo 2. Com isso o R2 ajustado apresentou melhora de 4,62\% para 6,27\%. O Modelo 3 apresenta significância estatística no nível de 5\%, com pvalor $=0,044068$. Assim, o último modelo é o que melhor elucida a variação do endividamento, mais precisamente, o Nível de capital de terceiros é NCT = -0,0270600 + 0,515694*I_TMC.

Como resultado, é possível constatar que o tempo médio no cargo dos gestores da equipe de alto escalão afeta positivamente a participação de capital de terceiros das companhias de energia elétrica da amostra. Neste contexto, não é possível aceitar a terceira hipótese de que o tempo médio no cargo está negativamente relacionado com o nível de capital de terceiros das empresas. De acordo com a literatura, o tempo do cargo da EGT pode representar um compromisso com o status quo e baixa criatividade (Hoffman et al., 2000). Por estarem mais comprometidos com a situação atual da empresa, o tempo no cargo é um indicativo de que gestores mais experientes no cargo de gestão tendem a aumentar o uso de recursos de terceiros em prol do capital próprio.

A primeira hipótese de que a idade média dos gestores está positivamente relacionada com o nível de capital de terceiros das empresas não foi significativamente apoiada. Acredita-se que a pequena variação da média de idade entre as equipes não tenha sido suficientemente correlacionada com a variação do nível de capital de terceiros das respectivas empresas. Ou seja, a amostra apresentou características mais homogêneas em relação a essa variável. Porém, verifica-se pelos Modelos 1 e 2 que a idade média está negativamente relacionada ao nível de capital de terceiros. Devido ao compromisso com o status quo, uma EGT mais antiga pode ser mais relutante em tentar novas ideias ou assumir riscos (Koufopoulos et al., 2008). Espera-se que os gerentes mais antigos estejam associados à estratégia de baixo custo mais orientada para a eficiência. Também é provável que os gerentes mais jovens enfatizem uma estratégia com um âmbito mais amplo, enquanto os gerentes mais velhos têm um escopo estratégico mais estreito (Goll et al., 2008).

A segunda hipótese a qual postula que o nível médio educacional está negativamente relacionado com o nível de capital de terceiros das empresas, não foi estatisticamente sustentada. 
Apesar disso, tal resultado admite a interpretação, com base no Modelo 1, de que o nível médio educacional da equipe é negativamente associado com a variável nível de capital de terceiros (endividamento) das companhias de energia elétrica. Os achados alcançados corroboraram com as ponderações de Bantel e Jackson (1989), Wiersema e Bantel (1992), e Barker e Mueller (2002) e contrário a Bortoluzzi et al. (2016).

\section{Conclusões}

Desde a publicação do trabalho seminal de Hambrick e Mason (1984), estudiosos ampliaram investigações sobre como as características da Equipe de Gestão de Topo (EGT) podem ser usadas para explicar a estratégia corporativa. Um pressuposto chave destas pesquisas é que as características observáveis são indicadores demográficos úteis de experiências individuais, habilidades, valores, estilos cognitivos e fontes de informação (Jehn, Northcraft \& Neale, 1999).

Diversos estudos concentraram-se em equipes de gestão de topo (EGT) e sua influência sobre decisões estratégicas. O efeito da EGT sobre o desempenho da empresa, em particular, tem sido de interesse contínuo para os pesquisadores de estratégia (Koufopoulos et al., 2008). Muitas variáveis como idade (Auden, Shackman, \& Onken, 2006), tempo no cargo (Carpenter, 2002) ou nível educacional (Herrmann \& Datta, 2005) têm sido utilizadas para analisar a composição das equipes.

Nessa linha, o propósito deste estudo foi relacionar as características da equipe do alto escalão e a estrutura de capital das empresas de energia listadas na Brasil, Bolsa, Balcão em 2018. A partir da análise estatística pode-se concluir que o tempo no cargo dos gestores da equipe do alto escalão tem uma relação positiva significativa com o nível de capitais de terceiros das empresas. Igualmente, uma maior experiência de um gestor no cargo pode torná-lo mais arrojado em relação ao endividamento da empresa.

Quanto às características idade média e nível educacional da equipe, verificou-se ausência de significância estatística. Apesar disso, ambas se mostraram negativas em relação a participação no capital de terceiros. Ou seja, quanto maior a média de idade e o nível educacional espera-se um menor nível de capital de terceiros na estrutura de capital da empresa.

A presente pesquisa possui algumas limitações, como por exemplo, a amostra, o período de análise e a quantidade de características gerenciais. Assim, os resultados encontrados não podem ser generalizados a outros grupos de empresas e períodos.

Recomenda-se que futuros estudos verifiquem a influência de outras características observáveis da equipe de gestão, como por exemplo, experiência internacional ou heterogeneidade de membros. Também, a relação dessas características com os diferentes níveis de capital próprio e de terceiros, de pesquisa e desenvolvimento e de investimentos das empresas. 


\section{Referências}

Acar, F. P. (2016) The effects of top management team composition on SME export performance: an upper echelons perspective. Central European Journal of Operations Research, 24, 833-852.

Auden, W. C., Shackman, J. D., \& Onken, M. H. (2006) Top management team, international risk management factor and firm performance. Team Performance Management, 12(7/8), 209-224.

Bantel, K. A., \& Jackson, S. E. (1989) Top management and innovations in banking: does the composition of the top management team make a difference? Strategic Management Journal, 10, 107-124.

Bany-Ariffin, A. N., McGowan, C. B., Júnior Tunde, M. B., \& Shahnaz, A. S. (2014) Top management characteristics and firm's international diversification activities: evidence from a Developing Nation. Pertanika Journal of Social Sciences \& Humanities, 22, 39-54.

Barker, V., \& Mueller, G. (2002) CEO characteristics and firm R\&D spending. Management Science, $v$. 48(6), 782-801.

Bell, S. T., Villado, A. J., Lukasik, M. A., Belau, L., \& Briggs, A. L. (2011) Getting specific about demographic diversity variable and team performance relationships: a meta-analysis. Journal of Management, 37(3), 709-743.

Bennouna, K., Meredith, G. G., \& Marchant, T. (2010) Improved capital budgeting decision making: evidence from Canada. Management Decision, v. 48(2), 225-247.

Bertrand, M., \& Schoar, A. (2003) Managing with style: The effect of managers on firm policies. The Quarterly Journal of Economics, 118(4), 1169-1208.

Bhattacharyya, S. C. (2007) Energy sector management issues: an overview. International Journal of Energy Sector Management,1(1).

Bortoluzzi, D. A., Zakaria, J., Santos, E. A., \& Lunkes, R. J. (2016) A influência das características dos executivos de alto escalão sobre a estrutura de capital: Um estudo em empresas listadas na BM\&FBOVESPA. Espacios, 37(37), 28.

Cabrera-Suárez, M. K., \& Martín-Santana, J. D. (2013) Top management teams and performance in non-listed family firms. Journal of Management \& Organization, v. 19, p. 405-423, 2013.

Camelo, C., Fernández-Alles, M., \& Hernández, A. B. (2010) Strategic consensus, top management teams, and innovation performance. International Journal of Manpower,31(6), 678-695.

Carpenter, M. A. (2002) The implications of strategy and social context for the relationship between top management team heterogeneity and firm performance. Strategic Management Journal, 23(3), 275-284.

Carpenter, M. A., \& Fredrickson, J. W. (2001) Top management teams, global strategic posture and the moderating role of uncertainty. Academy of Management Journal, 23, 275-284.

Carpenter, M. A., Geletkanycz, M. A., \& Sanders, G. W. (2004) Upper echelons research revisited: antecedents, elements, and consequences of top management team composition. Journal of Management, 30(6), 749-778. 
Chandler, A. (1962) Strategy and Structure: Chapters in the History of the Industrial Enterprise. Cambridge: MIT Press.

Chen, H.L., Hsu, W.-T., \& Huang, Y.-S. (2010) Top management team characteristics, R\&D investment and capital structure in the IT industry. Small Business Economics, 35, 319-333.

Chevalier, J. (1995) Capital structure and product market competition: Empirical evidence from the supermarket industry. American Economic Review, 85, 415-435.

Chuang, T. T., Nakatani, K., \& Zhou, D. An exploratory study of the extent of information technology adoption in SMEs: an application of upper echelon theory. Journal of Enterprise Information Management, v. 22, n. 1/2, p. 183-196, 2009.

Chuang, T. T., Nakatani, K., \& Zhou, D. (2007) The impact of managerial characteristics of top management team on the extent of information technology adoption: An exploratory study with the upper echelon theory. Anais do Americas Conference on Information Systems, Praga, 13.

Cyert, R. M., \& March, J. G. (1963) A behavioral theory of the firm. New Jersey: Prentice-Hall.

Damodaran, A. (2002) Investment evaluation: tools and techniques for determining the value of any asset. 3. ed. New York: John Wiley \& Sons, 2002.

Díaz-Fernández, M. C., González-Rodríguez, M. R., \& Pawlak, M. (2014) Top management demographic characteristics and company performance. Industrial Management and Data Systems, 114(3), 365386.

Díaz-Fernández, M. C., González-Rodríguez, M. R., \& Simonetti, B. (2015) Top Management Teams' demographic characteristics and their influence on strategic change, Quality \& Quantity, 49, 13051322.

Gitman, L. J. (2010) Principles of managerial finance (12a ed.). New Jersey: Prentice Hall.

Goll, I., Johnson, N. B., \& Rasheed, A. (2008) Top management team demographic characteristics, business strategy, and firm performance in the US airline industry - The role of managerial discretion. Management Decision, 46(2), 201-222.

Gu, J. (2008) An empirical study on the relation between characteristics of enterprise Top Management Team and strategic decision in industry cluster of China. International Journal of Human Resources Development and Management, 8(1/2), 96-110.

Guo, R. Liu, B.; Yang, \& Z.-L. (2011) Research on decision-making effectiveness factors of top management team. Anais do International Conference on Management Science \& Engineering, Roma, 18.

Hambrick, D. C. (2007) Upper echelons theory: an update. The Academy of Management Review, 32(2), 334-343.

Hambrick, D. C; \& Mason, P. A. (1984) Upper echelons: The organization as a reflection of its top managers. Academy of Management Review, 9, 193-206.

Herrmann, P., \& Datta, D. K. (2005) CEO experiences: Effects on the choice of FDI entry mode. Journal of Management Studies, 43(4), 755-778. 
Hoffman, J. J.; Williams, R. J.; Lamont, B. T.; \& Geiger, S. W. (2000) Managerial tenure and recovery following M-form reorganization in diversified firms. Journal of Business Research, 50, 287-296.

Hutzschenreuter, T., \& HorstkottE, J. (2013) Managerial services and complexity in a firm's expansion process: An empirical study of the impact on the growth of the firm. European Management Journal, 31, 137-151.

Jehn, K., Northcraft, G. B., \& Neale, M. A. (1999) Why differences make a difference: a field study of diversity, conflict and performance in workgroups. Administrative Science Quarterly, 44(4), 741-763.

Koufopoulos, D., Zoumbos, V., Argyropoulou, M., \& Motwani, J. (2008) Top management team and corporate performance: a study of Greek firms. Team Performance Management, v. 14(7/8), 340363.

Kor, Y. Y. (2006) Direct and interaction effects of top management team and board compositions on R\&D investment strategy. Strategic Management Journal, 27(11), 1081-1099.

Li, P. Y. (2016) The impact of the top management teams' knowledge and experience on strategic decisions and performance. Journal of Management and Organization, 22, 1-20.

Li, W., Zhang, S., \& Zhang, J. (2015) Top management team heterogeneity, diversification, and corporate performance: A panel smooth transition regression model. Romanian Journal of Economic Forecasting, 18(1), 129-142.

Maksimovic, V. (1998) Capital Structure in Repeated Oligopolies. Rand Journal of Economics, 19(3), 389-407.

Michel, J. G., \& Hambrick, D. C. (1992) Diversification posture and top management team characteristics. Academy of Management Journal, 35(1), 9-37.

Modigliani, F., \& Miller, M. H. (1958) The cost of capital, corporate finance and the theory of investment. American Economic Review, 48(3), 261-297.

Myers, S. C. (2001) Capital structure. Journal of Economic Perspectives, 15(2), 81-102.

Myers, S. C. (1984) The capital structure puzzle. Journal of Finance, 39(3), 575-92.

Myers, S. C., \& Majluf, N. S. (1984) Corporate financing and investment decisions when firms have information that investors do not have. Journal of Financial Economics, 13(2), p. 187-221.

Naranjo-Gil, D., Hartmann, F., Maas, V. S. (2008) Top management team heterogeneity, strategic change and operational performance. British Journal of Management, 19, 222-234.

O'Brien, J. P. (2003) The capital structure implications of pursuing a strategy of innovation. Strategic Management Journal, 24, 415-431.

Oppong, S. (2014) Upper Echelons Theory revisited: the need for a change from causal description to casual explanation. Management, 19(2), 169-183.

Pegels, C. C. Yang, B. (2000) Top management team impact on strategic assets accumulation capabilities. Management Decision, 38(10), 694-710. 
Pereira, V. L., Santana, A. C., Mendes, F. A. T., Khan, A. S. (2008) Análise do setor de energia elétrica do estado do Pará: uma aplicação da matriz de insumo-produto. Amazônia: Cência e Desenvolvimento, v. 4, n. 7.

Ross, S.A. (2007) Fundamentals of Corporate Finance, 4th ed., Sydney: McGraw Hill.

Wang, Y. K. (2015) The impact of prestigious top management team on international alliance formation: Evidence from Taiwanese electronics firms. Journal of Management \& Organization, 21(6), 835-852.

Wiersema, M. P., \& Bantel, K. A. (1992) Top management team demography and corporate strategic change. Academy of Management Journal, 35, 91-121.

Williamson, O. (1988) Corporate finance and corporate governance. Journal of Finance, 43, 567-591.

Xie, X. Y., Wang, W. L., \& Qi, Z. J. (2015) The effects of TMT faultline configuration on a firm's shortterm performance and innovation activities. Journal of Management \& Organization, 21(5), 558572. 